\title{
Sensing-Throughput Tradeoff for Cognitive Radios
}

\author{
Michele Guerrini, Luca Rugini, and Paolo Banelli \\ Department of Electronic and Information Engineering \\ University of Perugia \\ Perugia, Italy \\ \{luca.rugini, paolo.banelli\}@diei.unipg.it
}

\begin{abstract}
This paper focuses on sensing-throughput tradeoffs for overlay cognitive radio networks. The goal is to maximize the opportunistic secondary user throughput, by limiting the disturbance to primary user activity. Specifically, by assuming that a Poisson birth-death process characterizes the primary user traffic, the sensing-time bounds are imposed by granting enough accuracy to the detection of primary user activity, while the optimum duration for secondary user transmissions is obtained by maximizing analytical expressions for the secondary user average capacity. This paper also clarifies some subtle differences with the existing literature on the subject, which may lead to misleading results. Computer simulations corroborate the proposed theoretical findings.
\end{abstract}

Keywords-Cognitive radio networks; spectrum sensing; throughput.

\section{INTRODUCTION}

Cognitive radio (CR) is a promising technology for future communication standards aiming at exploiting frequency bands underutilized by primary legacy networks [1]. Among the different alternatives, overlay CR introduces a secondary network that opportunistically exploits the resources that are temporarily unused by primary users (PUs). To this end, secondary users (SUs) employ spectrum sensing [2], [3] in order to identify possible opportunities, followed by transmission periods if the PUs are detected as inactive.

Many CR approaches (e.g., [4]-[9]) assume that the SU activity periodically switches between a sensing operation and, if the PU is absent, a transmission phase. Longer sensing periods correspond to better detection accuracy of the PU presence, but also to a waste of transmitting opportunities for the SU. Furthermore, when the PU is detected as absent, the SU transmission period has to be carefully chosen. Indeed, too short transmission periods, followed by fixed-duration sensing periods, would lead to underutilization of the available opportunities, while too long transmission periods could generate too much interference to the PU, which may become active in the meanwhile. Thus, the problem of optimizing both the sensing duration and transmission duration of SU is of paramount importance for CR networks that want to both preserve the quality of PU transmissions and grant high-rate transmissions to SUs.

In this view, it is obviously useful to know the PU statistic in order to select the optimum sensing and transmission periods accordingly. A common assumption in [4]-[8] is to model the PU activity as a Poisson birth-death process, with independent traffic arrivals and durations. Exploiting this assumption, [4] and [5] have proposed an analytical framework to maximize the SU sensing efficiency, defined as the ratio between the transmission duration and the sensing-plus-transmission duration, under a constraint on the maximum amount of interference generated by the collisions of SU signals with PU signals. Intuitively, the maximization of the sensing efficiency should almost produce a maximum exploitation of the SU available capacity, i.e., a maximum throughput. Using the same assumption on the PU traffic model, [6] and [7] have directly maximized an approximated throughput of the SU, obtained by assuming that the PU may change activity status only once during the transmission period, under a constraint on the probability of collision [7] or on the maximum interference [6]. Sometimes, the design results of [6] and [7] are different from those of [4] and [5], and the throughput-optimal transmission period is shorter than the efficiency-optimal period. Since the throughput used in [6] and [7] is only an approximation, it is still unclear which of the two approaches is more convenient.

In this paper, we analytically assess the exact throughput, assuming that the PU may change activity status more than once during the transmission period. By means of this analysis, we reformulate the maximization of [6] and [7] using the exact throughput, with a different constraint on the interference generated by the SU. The results of our improved maximization show that the optimal transmission period is somewhat greater than that predicted by [6] and [7], and lower than that predicted by [4]. Moreover, by assuming that the PU may change activity status up to twice during the transmission period, we derive a novel analytical approximation of the throughput, which turns out to be more accurate than the approximation of [6] and [7]. In addition, we illustrate that the optimal transmission duration is quite sensitive to the maximization constraint, and hence we incorporate into the optimization problem also some practical bounds on the sensing period and on the transmission duration. Simulation results confirm the correctness of the obtained analytical throughput and validate the comparison among the proposed design and the designs of [4] and [7].

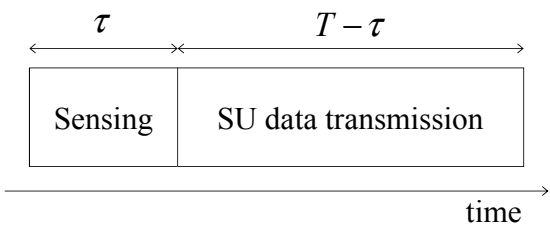

Fig. 1. Frame structure of the SU. 


\section{SYSTEM MODEL}

We consider a CR network where an SU tries to opportunistically access a given frequency band assigned to a licensed PU, whenever the PU is not using that band.

\section{A. PU Traffic Model}

We assume that the PU signal is characterized by exponentially distributed interarrivals, with mean duration $\beta_{1}$ of the busy (ON) state and mean duration $\beta_{0}$ of the idle (OFF) state. Therefore, the PU activity is modeled as a Poisson birth-death process with exponentially distributed ON-OFF periods. As a consequence, $\operatorname{Pr}\left\{H_{\alpha}\right\}=\beta_{\alpha} /\left(\beta_{0}+\beta_{1}\right)$ is the probability that the PU signal is present, for $\alpha=1$, or absent, for $\alpha=0$. In addition, we model the PU signal amplitudes as Gaussian distributed, which is valid, e.g., for multicarrier signals.

\section{B. SU Frame Structure}

As in [4]-[9], we assume that the SU adopts a periodic frame structure that alternates sensing periods and data transmission periods. We denote the frame duration with $T$. As shown in Fig. 1, at the beginning of the frame, the SU performs spectrum sensing, where $\tau$ is the sensing duration, in order to estimate the channel availability. Successively, if the outcome of the sensing operation is positive, the SU performs data transmission for the remainder time $T-\tau$ of the frame. In the successive frame, the SU must sense again the channel, in order to verify its availability before the next transmission.

\section{Spectrum Sensing}

To sense the presence or absence of the PU signal, we assume that the SU employs an energy detector (ED) with sampling frequency $f_{\mathrm{S}}$ and number of samples $N=\left\lfloor f_{\mathrm{S}} \tau\right\rfloor$. Although the PU traffic model is dynamic, we assume that the sensing duration $\tau$ is sufficiently short, so that the PU is permanently either ON or OFF during the whole sensing stage. The received signal, in the $H_{\alpha}$ hypothesis, with $\alpha \in\{0,1\}$, is modeled as $\mathbf{y}=\alpha \mathbf{x}+\mathbf{n}$, where $\mathbf{x}$ is the PU signal vector, assumed Gaussian with zero mean and covariance $\sigma_{\mathrm{x}}^{2} \mathbf{I}_{N}$, and $\mathbf{n}$ is the complex white Gaussian noise with zero mean and covariance $\sigma_{\mathrm{n}}^{2} \mathbf{I}_{N}$. The test statistic $\Lambda(\mathbf{y})=\|\mathbf{y}\|^{2}$ is compared with a threshold $\lambda$. This leads to a probability of false alarm [2] [3]

$$
P_{\mathrm{FA}}(\tau)=\operatorname{Pr}\left\{\Lambda(\mathbf{y})>\lambda \mid H_{0}\right\}=1-F_{2 N}\left(2 \lambda / \sigma_{\mathrm{n}}^{2}\right),
$$

where $F_{2 N}(x)$ is the cumulative distribution function (cdf) of a chi-squared random variable with $2 N$ degrees of freedom, and to a probability of detection [2] [3]

$$
P_{\mathrm{D}}(\tau)=\operatorname{Pr}\left\{\Lambda(\mathbf{y})>\lambda \mid H_{1}\right\}=1-F_{2 N}\left(\frac{2 \lambda}{\left(1+\gamma_{\mathrm{P}}\right) \sigma_{\mathrm{n}}^{2}}\right),
$$

where $\gamma_{\mathrm{P}}=\sigma_{\mathrm{x}}^{2} / \sigma_{\mathrm{n}}^{2}$ is the primary signal-to-noise ratio (SNR). Clearly, both (1) and (2) are function of the number of samples $N=\left\lfloor f_{\mathrm{S}} \tau\right\rfloor \approx f_{\mathrm{S}} \tau$ and hence of the sensing duration $\tau$.

\section{SENSING-ThroughPUT TRADEOFF}

First, we introduce the sensing-throughput tradeoff from an intuitive viewpoint. The basic idea is to optimize the sensing duration $\tau$ and the frame duration $T$ in order to maximize the throughput of the SU, with a constraint on the maximum inter- ference generated by the SU and seen by the PU in case of collision. As it will be explained later on, both $\tau$ and $T$ affect the probability of collision, and hence the throughput, which can be maximized with respect to the sensing time $\tau$ for a fixed frame duration $T$ [8], [9] or with respect to the frame duration $T$ for a fixed sensing time $\tau$ [6], [7].

Alternatively to the throughput approach [6]-[9], the sensing efficiency $\eta$ can be maximized, as expressed by [4], [5]

$$
\eta=\frac{T-\tau}{T}=1-\frac{\tau}{T}
$$

Indeed, from the SU perspective, it would be preferable to reduce the sensing duration $\tau$, and to increase the frame duration $T$, as much as possible, since the sensing duration represents a lost transmission opportunity. However, letting $\tau / T \rightarrow 0$, alone, does not guarantee that the interference seen by the PU is below a given threshold. Therefore, both maximization approaches (either throughput or sensing efficiency) require a constraint on the maximum interference caused by collisions.

Note that the maximum achievable throughput of the SU would be obtained for $\tau / T=0$, i.e., when the SU always transmits, without performing any sensing. If $\gamma_{S}$ denotes the SNR of the SU transmission, the maximum throughput achievable by the SU would be expressed as

$$
\begin{gathered}
C_{\text {max }}=\operatorname{Pr}\left\{H_{0}\right\} C_{0}+\operatorname{Pr}\left\{H_{1}\right\} C_{1}, \\
C_{0}=\log _{2}\left(1+\gamma_{\mathrm{S}}\right), \quad C_{1}=\log _{2}\left(1+\frac{\gamma_{\mathrm{S}}}{1+\gamma_{\mathrm{P}}}\right)
\end{gathered}
$$

Note that $C_{0}$ and $C_{1}$ in (5) are the conditional capacities when the PU is absent and present, respectively. Obviously, this always-transmit strategy does not try to avoid collisions between SU and PU signals, and hence the SU cannot control the interference sent from the SU to the PU. Therefore, a sensing strategy is necessary in order to reduce the collision events.

If the SU uses a sensing strategy, a collision with the PU signal still may happen, in two cases. In the first case, the PU signal is present, but the SU incorrectly miss-detects it and transmits data that overlap with the PU signal. In order to avoid collisions, the sensing time $\tau$ cannot be too short, otherwise the increased probability of miss detection $P_{\mathrm{MD}}(\tau)=1-P_{\mathrm{D}}(\tau)$ will increase the probability of collision. In the second case, a collision occurs when the PU signal is initially absent, and the SU correctly identifies its absence, but afterwards the PU becomes active during the SU data transmission phase. In order to avoid collisions, the frame duration $T$ cannot be too long, otherwise, even if the sensing outcome is correctly identified as $H_{0}$, there is an increased probability that the PU will change status and will become active during the SU transmission.

In this paper, we design the sensing time $\tau$ and the frame duration $T$ that maximize the throughput of the $\mathrm{SU}$, subject to a constraint on the probability of collision, or, more precisely, on the interference caused by the SU. In order to do this, in Section III.A we analytically derive the probability of collision and the average duration of collision, which enables the theoretical evaluation of the throughput in Section III.B.

\section{A. Probability of Collision versus Collision Duration}

Zarrin and Lim evaluate the probability of collision between an SU transmitted frame and the PU signal as [7] 
$P_{\mathrm{C}}(\tau, T)=\operatorname{Pr}\left\{H_{0}\right\}\left[1-P_{\mathrm{FA}}(\tau)\right] P_{C \mid H_{0}}(T-\tau)+\operatorname{Pr}\left\{H_{1}\right\} P_{\mathrm{MD}}(\tau),(6)$

where $P_{C \mid H_{0}}(T-\tau)=1-\exp \left(-(T-\tau) / \beta_{0}\right)$ is the probability that a silent PU changes status and becomes active within the SU transmission duration $T-\tau$. This is the exact probability of having at least one collision during the transmission duration $T-\tau$. Although exact, this probability does not consider that the collision duration may be shorter than $T-\tau$. For this reason, others, such as Pei et al., instead consider the ratio between the average duration of collision and the duration of the SU transmission [6]. We refer to this quantity as the collision duration ratio (CDR), although in [6] it is misnamed as probability of packet collision. The CDR is calculated as

$$
\begin{aligned}
\rho_{\mathrm{C}}(\tau, T)=\operatorname{Pr}\left\{H_{0}\right\}\left(1-P_{\mathrm{FA}}(\tau)\right) \rho_{C \mid H_{0}}(T-\tau)+ \\
+\operatorname{Pr}\left\{H_{1}\right\} P_{\mathrm{MD}}(\tau) \rho_{C \mid H_{1}}(T-\tau),
\end{aligned}
$$

where $\rho_{C \mid H_{f}}(T-\tau)$ is the conditional CDR on the transmission period, under the $H_{\alpha}$ hypothesis on the sensing period. With respect to the probability of collision (6), the CDR (7) is a better measure of the interference received by the PU, since the CDR takes into account that, even if there is a collision during a given frame, there may exist some OFF periods of the PU during the same frame. Similarly, Lee and Akyildiz consider the interference ratio $I=\rho_{\mathrm{C}}(\tau, T) / \operatorname{Pr}\left\{H_{1}\right\}$ [4], which is an approximated CDR for the PU, basically a scaled version of the $\mathrm{CDR}$ for the SU in (7). By exploiting some classic results on renewal process theory [10], we derive the conditional CDR as

$$
\rho_{C \mid H_{\alpha}}(T-\tau)=\operatorname{Pr}\left\{H_{1}\right\}+(-1)^{1-\alpha} \frac{\beta_{\alpha}\left[\operatorname{Pr}\left\{H_{1-\alpha}\right\}\right]^{2}}{T-\tau}\left(1-e^{-\frac{T-\tau}{\beta_{0} \operatorname{Pr}\left\{H_{1}\right\}}}\right)
$$

where $\alpha \in\{0,1\}$, which completes the theoretical CDR (7).

Note that the obtained CDR differs from the CDR results of [6], [7], [4]. Indeed, [6] and [7] assume that the frame duration $T$ is short, so that the PU can change its status only once within the SU transmission period $T-\tau$. This leads to [6], [7]

$$
\rho_{C \mid H_{\alpha}}^{(0 / 1)}(T-\tau)=(1-\alpha)+(-1)^{1-\alpha} \frac{\beta_{\alpha}}{T-\tau}\left[1-e^{-(T-\tau) / \beta_{\alpha}}\right] .
$$

Note that, under the $H_{0}$ hypothesis, $\rho_{C \mid H_{0}}^{(0 / 1)}(T-\tau)$ in (9) is an overestimation of $\rho_{C \mid H}(T-\tau)$ in (8), since assuming a single $\mathrm{PU}$ transition from OFF to ON neglects that the PU could go OFF again in the future. As it will be clear in the simulation section, this overestimation becomes inaccurate when the SU transmission duration $T-\tau$ increases. Dually, under the $H_{1}$ hypothesis, $\rho_{C \mid H_{1}}^{(0 / 1)}(T-\tau)$ is an underestimation of $\rho_{C \mid H_{1}}(T-\tau)$.

On the other hand, in [4], it is assumed that the frame duration $T$ is long, so that the PU always changes its status many times within the SU data transmission duration $T-\tau$, leading to the approximated conditional CDR result [4]

$$
\rho_{C \mid H_{\alpha}}^{(\infty)}(T-\tau)=\alpha+(-1)^{\alpha} \operatorname{Pr}\left\{H_{1-\alpha}\right\}\left[1-e^{-(T-\tau) / \beta_{\alpha}}\right] .
$$

The approximation (10) is accurate only for long $T-\tau$ and inaccurate for practical $T-\tau$, as it will be clear in Section IV.

In addition to the exact CDR calculation, this paper also presents a novel improved approximation for the conditional CDR. This improved approximation has been obtained by assuming that the PU can change its activity status up to twice within the SU data transmission duration $T-\tau$. We omit the derivation (with double integration over the statistics of the two possible state transition instants) and express the result as

$$
\begin{aligned}
& \rho_{C \mid H_{\alpha}}^{(0 / 1 / 2)}(T-\tau)= \\
& \quad=\alpha+(-1)^{\alpha} \frac{\beta_{1-\alpha}}{T-\tau}\left[1-\frac{\beta_{0} e^{-(T-\tau) / \beta_{0}}-\beta_{1} e^{-(T-\tau) / \beta_{1}}}{\beta_{0}-\beta_{1}}\right] .
\end{aligned}
$$

Differently from single-transition approximations [6] [7], the two-transition approximation (11) of the conditional CDR turns out to be quite accurate not only for short transmission durations $T-\tau$, but also for somewhat greater durations. Therefore, (11) may be useful in numerical optimization algorithms, for faster convergence with respect to (8).

\section{B. Throughput}

We analytically assess the achievable throughput $R(\tau, T)$ of the considered periodic sensing-transmission CR scheme. Since the SU transmission lasts $T-\tau$ and the whole frame duration is $T$, we express the throughput of the $\mathrm{SU}$ as

$$
\begin{aligned}
R(\tau, T) & =\frac{T-\tau}{T} \operatorname{Pr}\left\{H_{0}\right\}\left(1-P_{\mathrm{FA}}(\tau)\right) C_{H_{0}}(T-\tau)+ \\
& +\frac{T-\tau}{T} \operatorname{Pr}\left\{H_{1}\right\} P_{\mathrm{MD}}(\tau) C_{H_{1}}(T-\tau)
\end{aligned}
$$

where $C_{H_{\alpha}}(T-\tau)$ denotes the channel capacity of the SU conditioned on being in the $H_{\alpha}$ hypothesis during the sensing period. The conditional channel capacity is a weighted average of $C_{0}$ and $C_{1}$, depending on the absence or presence of the PU signal during the SU data transmission period. Therefore

$$
C_{H_{\alpha}}(T-\tau)=\left[1-\rho_{C \mid H_{\alpha}}(T-\tau)\right] C_{0}+\rho_{C \mid H_{\alpha}}(T-\tau) C_{1} .
$$

If we insert (13) into (12), using the conditional CDR result $\rho_{C \mid H_{\alpha}}(T-\tau)$ obtained in (8), and the capacity expressions in (5), we obtain the exact expression for the throughput $R(\tau, T)$, which is a main novel result of this paper.

Note that approximated throughput expressions have been previously derived in [6]-[9]. Some of these approximations can be obtained from (12)-(13) by replacing the exact conditional CDR (8) with the approximation (9), as detailed below.

- In [9], the throughput is obtained by assuming the PU as static during the whole frame. This means that the conditional CDR is set to zero under $H_{0}$ and to one under $H_{1}$, i.e., $\rho_{C \mid H_{\alpha}}(T-\tau)=\alpha$, which leads to $C_{H_{\alpha}}(T-\tau)=C_{\alpha}$. Using these approximations, the throughput (12)-(13) becomes equivalent to Equations 16, 17, and 18 in [9].

- In [7], the throughput is obtained by assuming a dynamic PU with, at maximum, a single state transition. This means that the conditional CDR in (8) is approximated with that in (9). With this change, the throughput (12)-(13) becomes equivalent to Equations from 18 to 22 in [7].

- In [6], the throughput is obtained by assuming at maximum a single transition, as in [7], but only the PU transition from OFF to ON is considered. This means that $\rho_{C \mid H_{0}}(T-\tau)$ is replaced by $\rho_{C \mid H_{0}}^{(0 / 1)}(T-\tau)$. Moreover, in [6] it is assumed that $C_{1}=0$ and that the sensing is perfect, i.e., $P_{\mathrm{FA}}(\tau)=P_{\mathrm{MD}}(\tau)=0$. By these assumptions, the throughput (12)-(13) becomes equivalent to Equations 1 and 8 in [6].

- In [8], the throughput is also obtained by assuming at maximum a single transition for the PU, but, additionally, [8] presents some simulation results obtained assuming up to two transitions for the PU signal, shown in Fig. 2 of [8]. The simulations of [8] reveal that, for $\operatorname{Pr}\left\{H_{0}\right\}=\operatorname{Pr}\left\{H_{1}\right\}$, there are only minor differences between the singletransition and the two-transition results. However, our si- 
mulations will demonstrate that, for $\operatorname{Pr}\left\{H_{0}\right\} \neq \operatorname{Pr}\left\{H_{1}\right\}$, a transmission period design based on the two-transition model is significantly different than that based on the single-transition model.

Other throughput expressions can be obtained from (12)(13) by convenient substitution of the exact conditional CDR (8) with suitable approximations, such as (11), derived in this paper assuming a maximum of two state transitions for the PU. Simulation results will show that the proposed approximation is more accurate than that in [7].

\section{Optimization of Sensing Duration and Frame Period}

We optimize the sensing duration $\tau$ and the frame period $T$ in such a way to maximize the throughput $R(\tau, T)$, with a constraint on the maximum CDR $\rho_{\mathrm{C}, \max }$, as expressed by

$$
\begin{aligned}
& \max _{\tau, T} R(\tau, T), \\
& \text { s.t. } \rho_{\mathrm{C}}(\tau, T) \leq \rho_{\mathrm{C}, \max } .
\end{aligned}
$$

Note that the CDR inequality (15) is not the only constraint that the time parameters $\tau$ and $T$ must satisfy. Indeed, in Section II.C, we have assumed that the probability of a PU state change during the sensing period $\tau$ is negligible, i.e.,

$$
P_{\text {change } \mid H_{\alpha}}(\tau)=1-\exp \left(-\tau / \beta_{\alpha}\right) \leq P_{\mathrm{S}},
$$

where $P_{\mathrm{S}}$ is chosen close to zero, leading to

$$
\tau \leq \tau_{\max }=-\min \left\{\beta_{0}, \beta_{1}\right\} \ln \left(1-P_{\mathrm{S}}\right) \approx \min \left\{\beta_{0}, \beta_{1}\right\} P_{\mathrm{S}} .
$$

Since the sensing duration $\tau$ affects both detection and false alarm performances, choosing a specific point of the receiver operating characteristic produces another constraint on $\tau$. If we want $P_{\mathrm{D}}(\tau) \geq P_{\mathrm{D}, \text { min }}$ and $P_{\mathrm{FA}}(\tau) \leq P_{\mathrm{FA}, \max }$, by approximating the chi-squared cdf in (1)-(2) with the Gaussian Q-function as

$$
F_{2 N}(x) \approx 1-Q\left(\frac{x}{2 \sqrt{N}}-\sqrt{N}\right),
$$

using $N \approx f_{\mathrm{S}} \tau$, and eliminating $\lambda$ in (1)-(2), we obtain

$$
\tau \geq \tau_{\min } \approx \frac{N_{\min }}{f_{\mathrm{S}}}=\frac{1}{f_{\mathrm{S}}}\left(\frac{Q^{-1}\left(P_{\mathrm{FA}, \max }\right)-\left(1+\gamma_{\mathrm{P}}\right) Q^{-1}\left(P_{\mathrm{D}, \min }\right)}{\gamma_{\mathrm{P}}}\right)^{2}
$$

In addition, we may desire that the probability that the PU changes state from OFF to $\mathrm{ON}$ within the frame duration $T$ does not exceed a given threshold $P_{\mathrm{T}}<1$, as expressed by

$$
P_{\text {change } \mid H_{0}}(T)=1-\exp \left(-T / \beta_{0}\right) \leq P_{\mathrm{T}} .
$$

Indeed, when the probability of a PU state change from OFF to $\mathrm{ON}$ during the frame is too high, a correct sensing outcome $H_{0}$ is invalid for the whole frame duration: as time increases, the sensing outcome becomes too outdated. By (20), we have

$$
T \leq T_{\max }=-\beta_{0} \ln \left(1-P_{\mathrm{T}}\right) .
$$

Consequently, the constrained maximization (14)-(15) should be solved in the domain identified by $\tau_{\min } \leq \tau \leq \tau_{\max }$ and $\tau<T \leq T_{\max }$, using (17), (19), and (21).

In order to solve (14)-(15), we may employ a hybrid method with exhaustive search over $\tau$ and analytical search over $T$. Specifically, for a given candidate $\bar{\tau}$ with $\tau_{\min } \leq \bar{\tau} \leq \tau_{\max }$, we analytically maximize $R(\bar{\tau}, T)$ over $T$, and denote the obtained frame duration with $T^{\prime}$. This analytical maximization is easy, since, for a wide range of parameters, $R(\bar{\tau}, T)$ is con- cave over $T$, Then, we consider (15): since $\rho_{\mathrm{C}}(\bar{\tau}, T)$ increases with $T$, we calculate $T_{C}$ such that $\rho_{\mathrm{C}}\left(\bar{\tau}, T_{C}\right)=\rho_{C \text {, max }}$. Thus, in correspondence of $\bar{\tau}$, we have the candidate frame duration $\bar{T}=\min \left\{T^{\prime}, T_{\mathrm{C}}, T_{\max }\right\}$, and the candidate maximum throughput $\bar{R}=R(\bar{\tau}, \bar{T})$. Finally, we repeat the above procedure for $\tau_{\min } \leq \bar{\tau} \leq \tau_{\max }$, and select the couple $(\bar{\tau}, \bar{T})$ that produces the maximum value of $\bar{R}$. The above maximization procedure can be simplified by reducing $P_{\mathrm{S}}$ up to $P_{\mathrm{S}}=\tau_{\min } / \min \left\{\beta_{0}, \beta_{1}\right\}$, in such a way that $\tau_{\max }=\tau_{\min }$, and performing the analytical search of $\bar{T}$ only for the unique $\bar{\tau}=\tau_{\min }$. In this case, keeping the sensing duration at minimum, we reduce both the SU notransmission time and the energy spent for spectrum sensing.

Now we compare the proposed throughput maximization (14)-(15) with [6]-[9]. In [8] and [9], $T$ is assumed fixed, and the throughput (for a static PU [9] or for a dynamic PU with a single transition [8]) is maximized versus $\tau$ and $\lambda$ with the constraint $P_{\mathrm{D}}(\tau, \lambda) \geq P_{\mathrm{D} \text {, min }}$. In [6] and [7], the sensing performances are fixed, i.e., $\tau$ and $\lambda$ are fixed, and the throughput (for a dynamic PU with a single transition) is maximized versus $T$, with a constraint on the probability of collision (6) [7] or on the CDR for the PU (interference ratio $I$ ) [6].

\section{SimUlation Results}

We first consider a case study with light PU load, where $\beta_{0}=950 \mathrm{~ms}$ and $\beta_{1}=50 \mathrm{~ms}$, so that $\operatorname{Pr}\left\{H_{1}\right\}=0.05$. Differently from [5] and [8], we assume a PU that does not change status during sensing. We fix $f_{\mathrm{S}}=10 \mathrm{kHz}$, and assume $\gamma_{\mathrm{P}}=3$ $\mathrm{dB}$ and $\gamma_{\mathrm{S}}=5 \mathrm{~dB}$. Using $P_{\mathrm{FA}, \max }=0.01$ and $P_{\mathrm{D} \text {, } \min }=0.9$, by (19), $\tau_{\text {min }} \approx 0.96 \mathrm{~ms}$, and, setting $P_{\mathrm{S}}=0.02$ in (17) leads to $\tau_{\max } \approx 1.01 \mathrm{~ms}$, so we have fixed the sensing duration to $\tau=\bar{\tau}=1 \mathrm{~ms}$. We also set $P_{\mathrm{T}}=0.5$ that gives $T \leq T_{\max } \approx 658$ $\mathrm{ms}$ from (21). Fig. 2 shows the normalized throughput, defined as $R(\bar{\tau}, T) / C_{\max }$, as a function of the frame duration $T$. For the approximated throughput approach of [7], if we omit the constraint on the probability of collision (6) (or on the CDR or on the interference ratio), the optimal frame duration would be $T^{\prime}=64 \mathrm{~ms}$; whereas, for the proposed approach, if we omit the CDR constraint (15), the optimal frame duration would be $T^{\prime}=205 \mathrm{~ms}$, i.e., significantly higher, although any $T \geq 100$ ms yields almost the same throughput of $T^{\prime}=205 \mathrm{~ms}$. Therefore, without CDR constraint (15), the proposed throughput approach could choose the same optimal value $T_{\max }=658 \mathrm{~ms}$ that is produced by the efficiency-based approach [4]. However, as clarified by Fig. 3, the CDR constraint (15) (or the constraint for the probability of collision) significantly affects the frame duration design. Indeed, in [7], the solution would be $\bar{T} \approx 64 \mathrm{~ms}$ only if the threshold on the maximum probability of collision (6) is greater than 0.065 , otherwise the optimal frame duration would be reduced. On the other hand, the CDR, which is the correct measure of the interference generated by the $\mathrm{SU}$, increases in a smoother way with respect to the probability of collision, and therefore the CDR constraint (15) used by our approach consents somewhat greater frame durations and hence greater efficiency. Fig. 3 also displays that, for practical frame durations, the multiple-transition approximation of the CDR obtained from the interference ratio of [4] is too inaccurate in this case, and becomes accurate only for greater frame durations, outside the considered range. Besides, Figs. 2-3 reveal that the proposed two-transition approximation accurately describes both the exact throughput and the exact CDR, unless 
the frame duration is too great. Specifically, Figs. 2-3 point out that, in this case, differently from [8], the optimal frame duration obtained from the two-transition model is significantly greater than that obtained from the single-transition model.

We now consider a second case study with moderate PU load, where $\beta_{0}=700 \mathrm{~ms}$ and $\beta_{1}=300 \mathrm{~ms}$, so $\operatorname{Pr}\left\{H_{1}\right\}=0.3$. We use the same parameters $f_{\mathrm{S}}, \gamma_{\mathrm{P}}, \gamma_{\mathrm{S}}, P_{\mathrm{FA}, \text { max }}, P_{\mathrm{D}, \text { min }}, P_{\mathrm{S}}$, and $P_{\mathrm{T}}$ as in the previous case, so that $\tau_{\min } \approx 0.96 \mathrm{~ms}$, $\tau_{\max } \approx 6.06 \mathrm{~ms}$, and $T_{\max } \approx 485 \mathrm{~ms}$. In this second case, for a fixed $\tau=\bar{\tau}$, the optimal throughput and the corresponding $T^{\prime}$ are somewhat similar for both our approach and [7]. Therefore in this case our approach produces results more similar to [7] rather than to [4]. Indeed, Fig. 4 exhibits the optimal transmission frame duration $T=\min \left\{T^{\prime}, T_{\max }\right\}$ for varying $\tau=\bar{\tau}$, omitting the CDR constraint. For both the proposed approach and [7], the maximum throughput in Fig. 4 is obtained when $\tau=1 \mathrm{~ms}$, which is also the optimal sensing time given by the efficiency-optimal approach of [4].

\section{CONCLUSION}

We have analytically evaluated the throughput of SUs in overlay CR networks, and we have performed an optimization of both the transmission period and the sensing time. The results of our optimization show that, depending on the system parameters, the optimal transmission period can be greater than that predicted in [7] and lower than that predicted in [4].

\section{ACKNOWLEDGMENT}

This research has been funded by the Fondazione Cassa di Risparmio di Perugia, within the project "Algoritmi cooperativi per reti wireless di sensori," with code 2010.011.0417.

\section{REFERENCES}

[1] S. Haykin, "Cognitive radio: brain-empowered wireless communications," IEEE J. Sel. Areas Commun., vol. 23, pp. 201-220, Feb. 2005.

[2] T. Yücek and H. Arslan, "A survey of spectrum sensing algorithms for cognitive radio applications," IEEE Commun. Surveys Tuts., vol. 11, pp. 116-130, First Quarter 2009.

[3] E. Axell, G. Leus, E. G. Larsson, and H. V. Poor, "Spectrum sensing for cognitive radio: state-of-the-art and recent advances," IEEE Signal Process. Mag., vol. 29, pp. 101-116, May 2012.

[4] W.-Y. Lee and I. F. Akyildiz, "Optimal spectrum sensing framework for cognitive radio networks," IEEE Trans. Wireless Commun., vol. 7, pp. 3845-3857, Oct. 2008.

[5] K. Chang and B. Senadji, "Spectrum sensing optimisation for dynamic primary user signal," IEEE Trans. Commun., vol. 60, pp. 3632-3640, Dec. 2012.

[6] Y. Pei, A. T. Hoang, and Y.-C. Liang, "Sensing-throughput tradeoff in cognitive radio networks: How frequently should spectrum sensing be carried out?," IEEE PIMRC 2007, Athens, Greece, Sept. 2007.

[7] S. Zarrin and T. J. Lim, "Throughput-sensing tradeoff of cognitive radio networks based on quickest sensing," IEEE ICC 2011, Kyoto, Japan, June 2011.

[8] L. Tang, Y. Chen, E. L. Hines, and M.-S. Alouini, "Effect of primary user traffic on sensing-throughput tradeoff for cognitive radios," IEEE Trans. Wireless Commun., vol. 10, pp. 1063-1068, Apr. 2011.

[9] Y.-C. Liang, Y. Zheng, E. C. Y. Peh, and A. T. Hoang, "Sensingthroughput tradeoff for cognitive radio networks," IEEE Trans. Wireless Commun., vol. 7, pp. 1326-1336, Apr. 2008.

[10] L. A. Baxter, "Some notes on availability theory," Microelectron. Reliab., vol. 25, pp. 921-926, 1985.

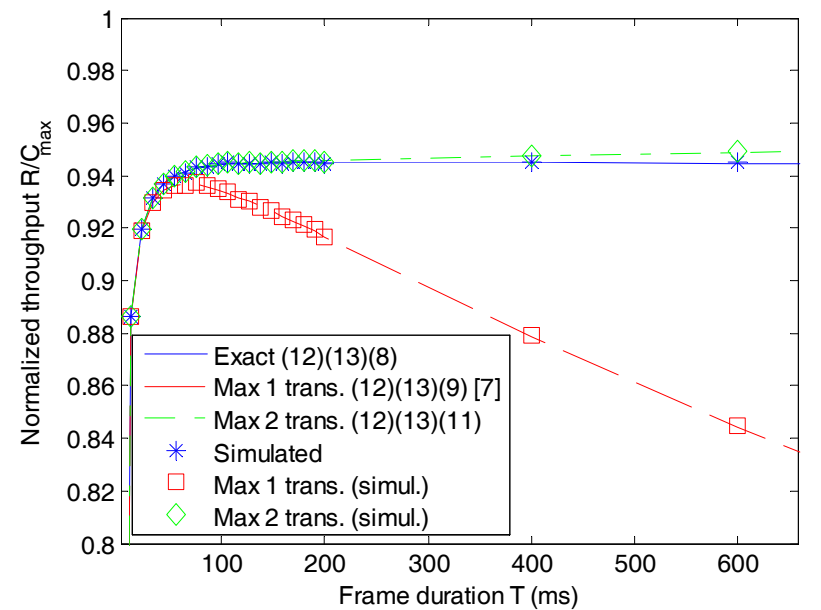

Fig. 2. First case study: normalized throughput versus the frame duration.

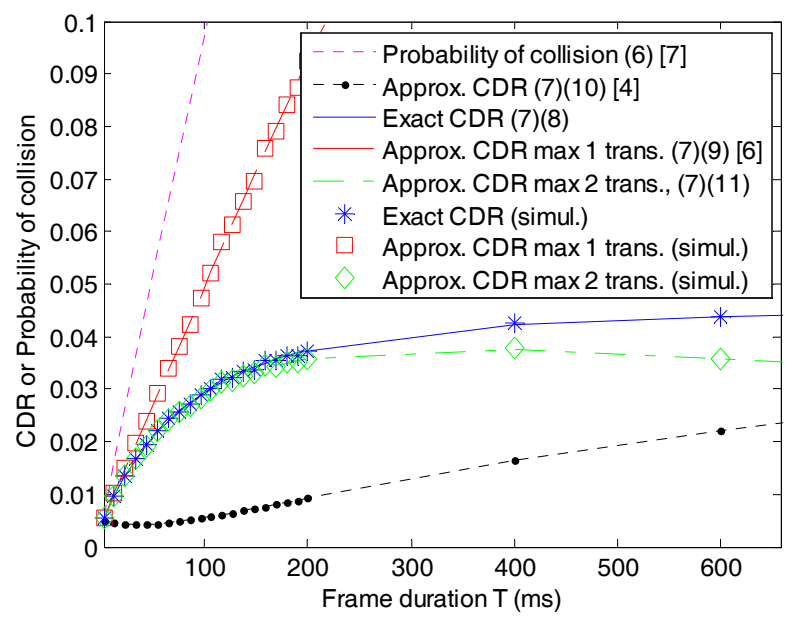

Fig. 3. First case study: collision duration ratio versus the frame duration.

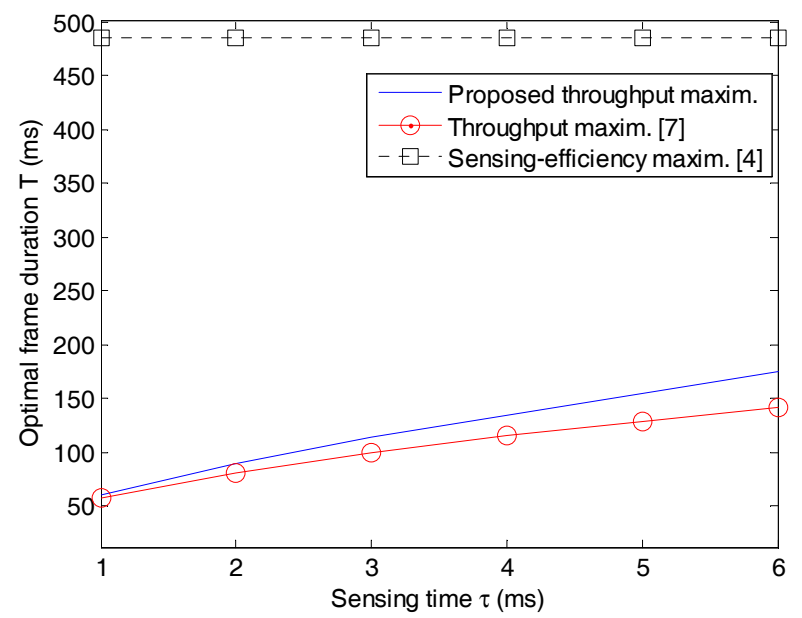

Fig. 4. Second case study: optimal frame duration versus the sensing time. 